\title{
Leitthema
}

Bundesgesundheitsbl 2015 · 58:730-737

DOI 10.1007/s00103-015-2168-8

Online publiziert: 10. Juni 2015

(c) Springer-Verlag Berlin Heidelberg 2015

Julia Sasse · H.R. Gelderblom

Robert Koch-Institut, Berlin, Deutschland

\section{Pockenausbrüche nach dem zweiten Weltkrieg in Deutschland}

zu. Daraus entwickeln sich pockenspezifische Läsionen an Haut und Schleimhäuten: Erytheme, Papeln, Bläschen, Pusteln bis hin zu Krusten im Abheilungsstadium. Bei etwa $30 \%$ der ungeimpften und $8 \%$ der geimpften Personen verlief die Pockenerkrankung tödlich. Klinisch steht die Abgrenzung gegenüber anderen fieberhaften Erkrankungen mit Hautbeteiligung, insbesondere den Windpocken, im Vordergrund. Die Differenzialdiagnose Pockenviren versus Herpesviren wurde auf dem bebrüteten Hühnerei und morphologisch (wesentlich schneller) durch Elektronenmikroskopie durchgeführt. Heute stehen neben der schnellen morphologischen Diagnostik ständig weiterentwickelte Nukleinsäure-basierte Verfahren zur Verfügung, die in wenigen Stunden eine Diagnose, aber auch eine Feintypisierung ermöglichen $[5,6]$.

Nach den Anthrax-Anschlägen im Jahr 2001 in den USA wurde aus Vorsorge auch in Deutschland gemeinsam von Bund, Ländern und Fachgesellschaften ein Rahmenkonzept ausgearbeitet, das die fachlichen und organisatorischen Vorbereitungen zu Diagnostik, seuchenhygienischen Maßnahmen, Organisation von Schutzimpfungen sowie zur Behandlung von Pocken aufzeigt.

Mit dem nachlassenden Wissen über diese Erkrankung in der Ärzteschaft steigt auch die Gefahr, dass die notwendige Expertise für das Management eines Ausbruchs verloren geht und ein - wenn auch als nicht sehr wahrscheinlich angesehener - Anschlag mit Pockenerregern große Auswirkungen auf das Gesundheitswesen haben könnte.

Die meisten Publikationen zur Kontrolle von Pockenausbrüchen stammen aus Afrika und Asien aus der Zeit der Eradikation. Die hier vorgelegte Auswertung aller deutschen Fälle aus der zweiten Hälfte des 20. Jahrhunderts soll dazu beitragen, auf die Problematik von Pockenerkrankungen aufmerksam zu machen, Expertise zu erhalten und gesicherte Verfahren zur Kontrolle eines Pockenausbruchs in einem Land mit einem hohen Standard des Gesundheitssystem zu entwickeln.

\section{Material und Methoden}

Es wurde eine Volltextsuche in den drei deutschen Hauptzeitschriften des Öffentlichen Gesundheitswesens „Deutsche Medizinische Wochenschrift", „Münchner Medizinische Wochenschrift“ und „Bundesgesundheitsblatt" für die Jahre 1945 bis 1975 durchgeführt. Da die Artikel zumeist online nicht recherchierbar sind, wurde die Suche von Hand durchgeführt. Ergänzend wurde über das „Deutsche Institut für Medizinische Dokumentation und Information" (DIMDI) eine Onlinerecherche durchgeführt.

\section{Ergebnisse}

\section{Überblick über die Ausbrüche}

Zwölf Pockenausbrüche sind in Deutschland zwischen 1947 und 1972 dokumentiert, 11 in der damaligen Bundesrepublik Deutschland und ein Ausbruch in der Deutschen Demokratischen Republik. Insgesamt erkrankten 95 Personen, von denen 10 verstarben; 81 Erkrankte waren geimpft.

1947 Wiesbaden (7 Erkrankte): Im Standortspital der US-Armee in Wiesbaden wurden an Pocken erkrankte US-Armeeangehörige behandelt. Zwei geimpfte deutsche Hilfspflegekräfte, bei denen keine Symptome bekannt wurden, infizierten ihre Mütter. Ein weiterer Pfleger entwickelte einen Ausschlag und infizier- 
te seine ihn mit Salbe behandelnde Vermieterin. Drei Mädchen aus dem Haus einer der geimpften Krankenhausmitarbeiterinnen erkrankten ebenfalls. Insgesamt erkrankten sechs geimpfte Personen leicht, ein vermutlich erfolglos geimpftes 6-jähriges Mädchen erkrankte schwer [7].

1957 Hamburg (1 Erkrankter): Ein 28-jähriger Techniker, der 1956 auf eine Wiederimpfung nur mit Knötchenbildung reagiert hatte [8], kehrte am 24.04.1957, einen Tag nachdem er Fieber und Halsschmerzen entwickelt hatte, mit dem Flugzeug aus Indien und $\mathrm{Pa}$ kistan zurück. In Hamburg angekommen, fuhr er mit einem Freund zu einem Restaurant. Die Nacht verbrachte er mit seiner Frau und seiner 8-jährigen Tochter in ihrer Ein-Zimmer-Wohnung, deren Badezimmer mit sechs weiteren Personen geteilt wurde [9]. Aufgrund des stark gestiegenen Fiebers begab er sich am 25.04. zu seinem Arzt, der eine Angina vermutete. Nach zwei Tagen entwickelte sich ein Erythem mit kleinen Knötchen. Am 29.04. wurde er mit der Diagnose „grippaler Infekt mit Ekzem“ stationär aufgenommen. Dabei teilte er das Zimmer noch einige Minuten mit einem anderen Patienten, bis der Verdacht auf Pocken gestellt und er isoliert wurde. Nach Bestätigung der Diagnose wurde der Krankenwagen gründlich desinfiziert. Zwischenzeitlich waren weitere 10 Patienten in dem Wagen transportiert worden [10], dennoch wurden keine weiteren Infektionen bekannt [9].

1958 Heidelberg (18 Erkrankte, 2 Todesfälle): Ein 38-jähriger Arzt, zuletzt 1943 wiedergeimpft, flog eine Woche nach Fieberbeginn von Indien nach Zürich. Am folgenden Tag, dem 05.12.1957 nahm er den Zug nach Heidelberg, wo er trotz Krankheitsgefühl am selben Tag die Arbeit aufnahm [11]. Aufgrund einer „Follikulitis“ und Halsentzündung suchte er am Abend die dermatologische Klinik auf. Dort wurde ein Pockenverdacht ausgesprochen und eine Isolation angeordnet. Der Patient hielt sich nachts im Isolierzimmer auf, arbeitete tagsüber aber in seinem Büro. Erst sieben Tage später wurden Pocken labordiagnostisch bestätigt [12].
Der Indexpatient hatte zehn seiner Kontakte angesteckt: sieben Krankenhausmitarbeiter, zwei Patienten und seine Haushälterin, die seine Kleidung gewaschen hatte [13]. In einigen Fällen beschränkte sich der Kontakt auf einen kurzen Aufenthalt im selben Zimmer. Darüber hinaus ist es zu sieben Sekundärinfektionen im Krankenhaus gekommen. Während des Ausbruchs sind zwei Personen verstorben: eine 26-jährige Ärztin, die aufgrund einer Dermatitis in der Kindheit nicht geimpft worden war, und eine 70-jährige Patientin mit Leberzirrhose. Alle anderen Infizierten waren geimpft und wiesen nur einen milden Krankheitsverlauf auf [12]. Der Indexpatient wurde in einem späteren Verfahren der fahrlässigen Körperverletzung für schuldig befunden [14].

1959 Berlin (Ost) (1 Erkrankter): Ein 27-jähriger Student, der nach eigenen Angaben bis 1958 jedes Jahr wiedergeimpft wurde, allerdings seit 1953 nur mit einer minimalen Hautreaktion, kehrte am 03.04.1959 über Prag, wo er Fieber entwickelte, aus Indien zurück. Am nächsten Tag flog er von Prag nach Berlin, wo er von einem Freund in einem Auto mitgenommen wurde [15]. Am 05.04. suchte er einen Arzt auf, der ihn in ein Krankenhaus überwies. Dort wurde eine Pockeninfektion vermutet und zwei Tage später elektronenoptisch bestätigt [13]. Es wurden keine Übertragungen gemeldet.

1961 Ansbach (4 Erkrankte, 1 Todesfall): Eine Woche nach seiner Rückkehr aus Indien entwickelte ein 28-jähriger Fotograf am 18.03.1961 unspezifische Symptome mit Fieber [16]. Er hatte zwei Erstimpfnarben und war 1958 ohne Erfolg wiedergeimpft worden. Am 23.03. bekam er "rote Fleckchen“ mit Bläschen im Mund. Unter Windpockenverdacht wurde er am 25.03. isoliert. Aus der Klinik schrieb er seinem Freund, der mit ihm durch Indien gereist war. Erst dessen Arzt vermutete eine Pockeninfektion und informierte das Gesundheitsamt [10]. Daraufhin wurde der Patient erneut untersucht und die Pockeninfektion am 29.03. bestätigt.

Die Eltern des Indexpatienten, die vom 18.03. bis 25.03. mit ihm Kontakt hatten, waren in der Kindheit geimpft und wurden am 31.03. wiedergeimpft. Die Mutter zeigte am 04.04. erste Symptome, der Vater am 07.04. [16]. Während der Vater überlebte, verstarb die Mutter an hämorrhagischen Pocken. Der Arzt der Isolierstation, der am 29.03. wiedergeimpft worden war und aufgrund ausbleibender Impfreaktion vier Tage später erneut geimpft wurde, aber nur mit einer Knötchenbildung reagierte, erkrankte am 22.04. mittelschwer an Pocken [16].

1961/62 Düsseldorf (5 Erkrankte, 2 Todesfälle): Zehn Tage nach seiner Rückkehr aus Liberia, am 13.12.1961, entwickelte ein 37-jähriger Ingenieur Fieber. Er war in seiner Kindheit geimpft und 1954 und 1959 mit einer kleinen Pustelreaktion wiedergeimpft worden [17]. Am 15.12. suchte er einen Arzt auf, der angesichts einer Fehlinformation über das Reisedatum eine Rachenentzündung annahm. Am 17.12. entwickelte sich ein als Akne gedeutetes Erythem, woraufhin der Patient am 19.12. seine Büroarbeit wieder aufnahm.

Am 29.12. erkrankte sein ohne Erfolg erstgeimpfter 5-jähriger Sohn, auf dessen Wiederimpfung aufgrund eines aufflackernden Ekzems verzichtet worden war. Als zwei Tage später auch die in der Kindheit geimpfte Ehefrau erste Symptome zeigte, wurde am 01.01.1962 die Verdachtsdiagnose Pocken gestellt: Alle drei Personen sowie die drei weiteren Kinder der Familie und das Dienstmädchen wurden im Krankenhaus isoliert [18]. Die im siebten Monat schwangere Frau erkrankte nur leicht und gebar ein gesundes Baby. Die Erkrankung des 5-Jährigen wies einen mittelschweren Verlauf auf [17].

Am 10.01.1962 trat bei einer Krankenschwester der Isolierstation Müdigkeit und zwei Tage später ein rapider Fieberanstieg auf. Sie verstarb am 20.01. an hämorrhagischen Pocken. In ihrer Kindheit war sie geimpft und bei der Aufnahme der Patienten am 01.01.1962 ohne Erfolg wiedergeimpft worden. Auf eine weitere Impfung sechs Tage später reagierte sie mit Knötchenbildung [17]. Eine zweite Krankenschwester, die ohne direkten Kontakt mit den Erkrankten im „Schleusendienst" arbeitete, erkrankte am 01.02, sie verstarb am 17.02. an sekundär-hämorrhagischen Pocken. Auch sie war am 
Tag der Patientenaufnahme am 01.01.1962 - nur mit Knötchenreaktion - wiedergeimpft worden [17].

1962 Monschau-Simmerath (34 Erkrankte, 1 Todesfall): Dreizehn Tage nach seiner Rückkehr aus Indien entwickelte ein 32-jähriger Mechaniker am 05.01.1962 fieberhaftes Unwohlsein. Als drei Tage später einzelne Hautbläschen auftraten, diagnostizierte der Hausarzt Windpocken. Am 21.01. kehrte der Indexpatient zur Arbeit zurück. Seine 9-jährige Tochter, die einmal ohne Erfolg geimpft worden war, erkrankte am selben Tag. Am 31.01. wurde der Pockenverdacht gestellt und das Kind in ein Krankenhaus der nächstgelegenen Großstadt gebracht, da der diensthabende Arzt die Aufnahme zugesagt hatte. Nach Einschalten der zuständigen mittleren und obersten Gesundheitsbehörden wurde das Mädchen stattdessen in einem Kreiskrankenhaus in Wohnortnähe isoliert [18]. Die Einschleusung in das Einzelzimmer, das eine Doppeltürenschleuse zum Flur und eine eigene Toilette besaß, erfolgte von außen. Alle fünf weiteren Räume der Isolierstation waren geräumt. Dennoch erkrankten weitere zwölf Personen in der Klinik, mehrere ohne direkten Kontakt zur Erkrankten. Vier von ihnen infizierten insgesamt sieben weitere Personen, von denen wiederum drei jeweils eine weitere Person ansteckten. Der Indexpatient infizierte neben der Tochter auch seine Ehefrau und den erstgeimpften 7-jährigen Sohn. Durch das Mädchen wurden auch die im selben Haus wohnende Großmutter, der Hausarzt, ein Arzt aus Aachen, der Krankenwagenfahrer sowie vier Nachbarn angesteckt, die alle geimpft waren und nur leicht erkrankten.

Eine 32-jährige, in der Kindheit geimpfte Patientin des Krankenhauses verstarb an hämorrhagischen Pocken. Einige Personen mit unspezifischen Symptomen, die zumeist in ihrer Kindheit geimpft worden waren, konnten erst dadurch als Pockenvirus-infiziert identifiziert werden, dass sie Kontaktpersonen infizierten. Bei einer erkrankten Person konnte der Überträger nicht ermittelt werden [18].

1965 Kulmbach (1 Erkrankter): Ein 49-jähriger Techniker klagte einen Tag nach seiner Rückkehr aus Ostafrika, wo

Bundesgesundheitsbl 2015 · 58:730-737 DOI 10.1007/s00103-015-2168-8

(c) Springer-Verlag Berlin Heidelberg 2015

\section{J. Sasse $\cdot$ H.R. Gelderblom}

\section{Pockenausbrüche nach dem zweiten Weltkrieg in Deutschland}

\section{Zusammenfassung}

Hintergrund. Seit 1980 gelten die Pocken als ausgerottet. Es ist jedoch nicht auszuschließen, dass der Erreger der Pocken, das Variola-Virus, aus nicht vernichteten oder aus nach der Eradikation nicht an eines der beiden WHO-Referenzzentren (WHO Collaborating Smallpox Laboratories) abgegebenen Stämmen zu bioterroristischen Anschlägen genutzt wird. Aufgrund der Weiterentwicklung des molekularen Engineerings steigt zudem die Gefahr der Erzeugung neuer Pockenviren.

Ziel der Arbeit. Die Auswertung von klinischen und epidemiologischen Daten, aus denen Schlussfolgerungen für die Ausbruchskontrolle dieser lebensbedrohlichen Krankheit abgeleitet werden.

Methoden. Es handelt sich hier um eine Volltext-Handrecherche in den Hauptpublikationsorganen des öffentlichen Gesundheitswesens in Deutschland von 1945 bis 1975.

Ergebnis und Diskussion. Nach dem zweiten Weltkrieg ereigneten sich in Deutschland zwölf Pockenausbrüche, die hier hinsichtlich folgender Kenndaten ausgewertet werden: Beginn der Kontagiosität, Schutzwirkung der Impfung, Booster-Effekt bei der Wiederimp- fung und Ansteckungsorte. Insgesamt erkrankten 95 Personen, von denen 10 verstarben. 81 der erkrankten Personen waren geimpft und entwickelten zu $91 \%$ nur leichte Symptome. Leicht Erkrankte stellten nach primärer Fehldiagnose durch ihre weitere Teilnahme am öffentlichen Leben ein besonderes Risiko für die Weiterverbreitung des Erregers dar. Die hier dargestellten Daten zeigen ein niedriges Infektionsrisiko für die ersten drei Krankheitstage, wodurch antiepidemische Maßnahmen und die Kontaktverfolgung erleichtert werden. Die meisten Infektionen fanden im Krankenhaus statt, dies unterstreicht die Bedeutung einer guten Vorbereitung auch von peripheren Krankenhäusern. Die hier dargestellten Daten dokumentieren die Erfahrungen aus Deutschland und sollten zur Planung medizinischer und epidemiologischer Maßnahmen zur Ausbruchskontrolle hinzugezogen werden.

\section{Schlüsselwörter}

Pocken · Wiederimpfung · BoosterEffekt · Antiepidemische Maßnahmen . Vorsorgeplanung

\section{Lessons learnt from the German smallpox outbreaks after World War II}

\section{Abstract}

Background. Even though smallpox was declared eradicated by WHO in 1980, it cannot be ruled out that the etiological variola virus could be used as a biological weapon. Undestroyed viruses from biowarfare programmes, virus strains left undetected in a freezer or dangerous recombinant poxvirus constructs could cause dangerous outbreaks in a relatively unprotected population.

Objectives. Despite an abundance of studies performed during the eradication of smallpox, epidemiological data for preparedness planning and outbreak control in modern, industrialized countries are scarce.

Material and methods. Full-text hand search for the period from 1945 to 1975 in the main German public health journals. Results. After World War II 12 smallpox outbreaks occurred in Germany. They were studied with the focus on the period of contagiousness, the protective effect of vaccination, booster-effect of revaccination and the place of infection. A total of 95 individu- als contracted smallpox, including 10 fatalities. Despite having been previously vaccinated, 81 vaccinated persons came down with smallpox, yet $91 \%$ of them developed only mild symptoms. These patients presented a high risk for spreading the infection to contact persons due to misinterpretation of symptoms and the continuing social contacts. Basically, the risk of transmission in the first 2 to 3 days after onset of symptoms was low, thus facilitating antiepidemic measures. The importance of hospital preparedness is emphasized by the fact that most infections occurred in hospitals.

Conclusion. The data analyzed provide valuable information for today's outbreak response planning and counter bioterrorism preparedness.

Keywords

Smallpox - Vaccination · Transmission . Antiepidemic measures Preparedness 
Tab. 1 Zahl der Infizierten pro Pockenausbruch; Impfstatus und Beginn der Isolierung der Indexpatienten. (sortiert nach der Anzahl infizierter Personen durch den Indexpatienten)

\begin{tabular}{|c|c|c|c|c|c|c|}
\hline \multirow[t]{2}{*}{ Ausbruche } & \multicolumn{2}{|c|}{$\begin{array}{l}\text { Infizierte Kontakte (verstorbene } \\
\text { Kontakte) }\end{array}$} & \multirow[t]{2}{*}{$\begin{array}{l}\text { Letzte erfolgreiche Impfung } \\
\text { des Indexpatienten (Jahre) }\end{array}$} & \multicolumn{3}{|c|}{$\begin{array}{l}\text { Zeit (Tage) zwischen dem Auftreten erster Symptomen } \\
\text { und }\end{array}$} \\
\hline & $\begin{array}{l}\text { durch Index- pa- } \\
\text { tient }\end{array}$ & $\begin{array}{l}\text { während des } \\
\text { Ausbruchs }\end{array}$ & & $\begin{array}{l}\text { Aufsuchen eines } \\
\text { Arztes }\end{array}$ & $\begin{array}{l}\text { Verdachts- } \\
\text { (bestätigte) } \\
\text { Diagnose }\end{array}$ & Isolation \\
\hline Hannover 1967 & 0 & 0 & $<1$ & 1 & $1(1)$ & n.b. ${ }^{a}$ \\
\hline Berlin (East) 1959 & 0 & 0 & 1 & 2 & $2(4)$ & 2 \\
\hline Hamburg 1957 & 0 & 0 & $16^{\mathrm{b}}$ & 2 & $6(7)$ & 6 \\
\hline Hannover 1972 & 0 & 0 & $\sim 22$ & 1 & $5(5)$ & 3 \\
\hline Kulmbach 1965 & 0 & 0 & $37^{b}$ & 4 & $4(5)$ & 4 \\
\hline Regensburg 1967 & 1 & 1 & 56 & 2 & $6(6)$ & 6 \\
\hline Ansbach 1961 & $2(1)$ & $3(1)$ & $\sim 26$ & 2 & $9(10)$ & 7 \\
\hline Düsseldorf 1961/62 & 2 & $4(2)$ & 2 & 2 & $18(19)$ & $18(1)^{d}$ \\
\hline Monschau 1962 & 3 & $33(1)$ & n.b. ${ }^{a}$ & 3 & $26(26)$ & $26^{d}$ \\
\hline Heidelberg 1958 & $10(2)$ & $17(2)$ & 15 & 8 & $8(15)$ & 8 \\
\hline Meschede 1970 & $17(3)$ & $19(4)$ & $--^{c}$ & 1 & $6(6)$ & $1(5)^{d}$ \\
\hline \multicolumn{7}{|c|}{$\begin{array}{l}\text { an.b. nicht bekannt. } \\
\text { b }<1 \text { Jahr vorher eine Wiederimpfung mit Knötchenreaktion. } \\
\text { c }<1 \text { Jahr vorher eine „Impfung“ mit Vaccinia-Antigen. } \\
\text { dIn Klammern die Anzahl der Tage mit unzureichender Isolierung. }\end{array}$} \\
\hline
\end{tabular}

Tab. 2 Zusammenhang zwischen Impfstatus und Schwere der Pockenerkrankung

\begin{tabular}{|c|c|c|c|c|}
\hline Impfstatus und Erkrankung & Erkrankte $^{\mathrm{a}}$ & Todesfälle & Fälle (\%) & $\begin{array}{l}\text { Todesfälle } \\
\text { (\%) }\end{array}$ \\
\hline Geimpft, milde Erkrankung & 75 & $3^{b}$ & \multirow[t]{2}{*}{86,3} & \multirow[t]{2}{*}{8,5} \\
\hline $\begin{array}{l}\text { Geimpft, (mittel-)schwere Er- } \\
\text { krankung }\end{array}$ & 7 & 4 & & \\
\hline $\begin{array}{l}\text { Nicht geimpft, milde Erkran- } \\
\text { kung }\end{array}$ & 0 & 0 & \multirow[t]{2}{*}{8,4} & \multirow[t]{2}{*}{25} \\
\hline $\begin{array}{l}\text { Nicht geimpft, (mittel-)schwere } \\
\text { Erkrankung }\end{array}$ & 8 & 2 & & \\
\hline Unbekannter Impfstatus & 5 & 1 & 5,3 & 20 \\
\hline Summe & 95 & 10 & 100 & 10,5 \\
\hline
\end{tabular}

er in einem Missionskrankenhaus neben einem Zimmer mit Pockenkranken genächtigt hatte, über Gliederschmerzen und Husten, die sich vier Tage später, am 23.10.1965, verstärkten. Er war insgesamt sechs Mal gegen Pocken geimpft worden, auf die letzten drei Impfungen zeigte er eine Knötchenreaktion. Am 24.10. rief er wegen Fiebers einen Arzt, ging aber am nächsten Tag wieder arbeiten, da er sich besser fühlte. Als sich am 27.10. vereinzelte makulöse Erytheme zeigten, die sich am nächsten Tag zu Vesikeln und Pusteln entwickelten, wurde eine Pockenerkrankung vermutet. Der Patient wurde am 28.10. isoliert und die Diagnose nach wenigen Stunden elektronenmikroskopisch bestätigt [19]. Es traten keine Übertragungen der Pocken auf [20].

1967 Hannover (1 Erkrankter): Ein Hautarzt hatte auf einer Indienreise zwei Krankenhäuser für Pockenkranke besucht. Einen Tag nach seiner Rückkehr entwickelte er Vesikeln auf den Lippen, am Körper und unter den Fußsohlen. Er vermutete sofort eine Pockeninfektion, die auch am selben Tag, am 24.03.1967, bestätigt wurde. Er war 1966 und 1967 wiedergeimpft worden. Es wurden keine Infektionen durch den Indexpatienten bekannt [21].
1967 Regensburg (2 Erkrankte): Eine 58-jährige Geschäftsfrau bekam 12 Tage nach ihrer Rückkehr aus Indien, am 04.03.1967, Kopfschmerzen. Wegen einer Hautrötung wurde ihr am 06.03. Penicillin gespritzt. Sie war als Kind erfolgreich erstgeimpft worden, die Wiederimpfung 1965 war nicht kontrolliert worden und es war keine weitere Impfnarbe sichtbar. Scharlachförmige Effloreszenzen deutete der Hausarzt als allergische Reaktion auf das Antibiotikum. Am 10.03. verschlechterte sich ihr Zustand, und sie wurde daraufhin unter der klinischen Diagnose „sekundär-hämorrhagische Pocken“ im Krankenhaus isoliert.

Am gleichen Tag wurde ihre im selben Haus wohnende 84-jährige Tante, die seit ihrer Kindheit an Herzinsuffizienz litt, mit Vaccinia-Antigen und hohen Dosen Immunglobulin prophylaktisch behandelt. Sie erkrankte am 23.03. an einer milden Form [22].

1970 Meschede (20 Erkrankte, 4 Todesfälle): Ein 20-jähriger ungeimpfter Elektriker reiste, nachdem er Vaccinia-Antigen erhalten hatte, nach Pakistan. Während der Reise wurde er erfolglos geimpft. Am 11.01.1970, zehn Tage nach seiner Rückkehr, wurde er mit Fieber als Typhusverdacht stationär in einem Einzelzimmer 
isoliert. Drei Tage später, am 14.01.1970 wurde klinisch der Verdacht auf Pocken gestellt. Nach der labordiagnostischen Bestätigung am 16.01. wurde der Patient in ein Krankenhaus mit höherer Isolierleistung verlegt. Dort erfolgten keine Übertragungen.

Im ersten Krankenhaus erkrankten jedoch 13 Patienten sowie drei Krankenschwestern und ein Besucher, wahrscheinlich durch Übertragungen des Erregers aufgrund von Luftzug $[1,23,24]$. Drei vorher ungeimpfte Personen erkrankten sehr schwer, eine von ihnen, eine 17-jährige Krankenschwester, verstarb. Die anderen Infizierten waren geimpft [25] und erkrankten nur leicht. Trotz Impfung und milder Pockensymptome verstarben auch drei ältere Patienten mit schweren Grunderkrankungen. Zusätzlich traten zwei Sekundärinfektionen durch Krankenhauspatienten auf [24].

1972 Hannover (1 Erkrankter): Ein 24-jähriger Handwerker entwickelte $11 \mathrm{Ta}$ ge nach seiner Rückkehr aus dem Kosovo unspezifische Symptome. Am nächsten Tag, dem 23.03.1972, bildeten sich kleine Vesikeln im Gesicht. Der aufgesuchte Dermatologe verschrieb eine Heilsalbe. Als sich der Ausschlag zwei Tage später über den ganzen Körper ausbreitete, wurde der Patient mit Windpockenverdacht hospitalisiert. Am 27.03. zeigte er über den Körper verteilte Vesikeln. Der klinische Verdacht auf Pocken wurde noch am selben Tag durch Elektronenmikroskopie bestätigt [20].

Der Patient teilte sich in Hannover zusammen mit sechs weiteren Personen ein Hostelzimmer [26]. Es traten keine weiteren Fälle auf. Eine besondere Herausforderung für die Anamnese und Diagnostik ergab sich durch einen parallel auftretenden Windpockenausbruch [27].

\section{Diskussion}

\section{Beginn der Kontagiosität bei Pocken}

Nach Aussage der WHO wird ein Erkrankter erst nach dem Auftreten von Fieber infektiös [28]. Die Analyse der 12 Ausbrüche in Deutschland unterstreicht diese für das Management der Erkran- kung günstige Feststellung. Indexpatienten, die innerhalb von zwei bis vier Tagen nach dem ersten Auftreten von Fieber effizient isoliert wurden, haben keine weitere Person angesteckt ( $\bullet$ Tab. 1). Erfolgte die Isolierung erst fünf Tage nach Fieberbeginn, d. h. beim Auftreten der pathognomonischen Vesikeln, nahm das Risiko einer Übertragung zu.

Diese Ergebnisse stimmen mit denen aus Untersuchungen aus der Zeit der Ausrottungskampagne überein. Der zweite Tag nach Fieberbeginn wird als der Tag beschrieben, an dem die Kontagiosität beginnt [29]. Die höchsten Viruskonzentrationen im Rachenspülwasser mit Titern von $10^{5} \mathrm{pfu} / \mathrm{ml}$ wurden am zweiten bis vierten Tag nach Fieberbeginn gemessen [30]. Eine andere Untersuchung an $119 \mathrm{~Pa}-$ tienten ergab Virus-negative Rachenspülwasser an den ersten beiden Tagen nach Fieberbeginn. Am dritten Tag waren 50\% der Proben positiv, gefolgt von Tag sechs bis neun mit einem Maximum von $65 \%$ positiven Proben [31].

Studien aus Indien mit Haushaltsangehörigen von Erkrankten zeigten jedoch, dass sich bei $10 \%$ der asymptomatischen Kontaktpersonen (34/328 bzw. 5/51) Virus im Rachenspülwasser nachweisen ließ, die Titer lagen allerdings durchschnittlich zwei Log-Stufen niedriger als bei Pockenpatienten [32-35]. Nur vier respektive eine dieser positiv getesteten Kontakte erkrankten anschließend an Pocken [30]. Die Hälfte der positiv getesteten Kontakte war geimpft. Alle Indexpatienten von positiv getesteten Kontaktpersonen zeigten sehr schwere, teilweise hämorrhagische Verläufe. Bei allen Indexpatienten mit mildem Verlauf blieben die Haushaltskontakte Virus-negativ [29]. Nach diesen Studien kann eine Übertragung durch asymptomatische Kontaktpersonen - insbesondere von Personen mit Kontakt zu schwer erkrankten Patienten - nicht ausgeschlossen werden und bedarf eines genauen Augenmerks. In Deutschland könnte der Fall aus Wiesbaden im Jahre 1947 auf ,asymptomatischer Übertragung" beruhen, wobei aber auch die Übertragung durch kontaminierte Wäsche in Betracht gezogen werden sollte, wie bei der Erkrankung der Haushaltshilfe des infizierten Arztes in Heidelberg im Jahre 1958 [13], zumal Pockenvi- ren in Krusten längere Zeit infektiös bleiben [36].

\section{Der Einfluss von Impf- und Gesundheitsstatus auf die Schwere der Pockenerkrankung}

Von den 81 geimpften und dennoch an Pocken erkrankten Personen hatten $90 \%$ einen milden oder sehr milden Verlauf, selbst wenn ihre Impfungen in der Kindheit erfolgten und daher sehr lange zurücklagen. Sieben geimpfte Personen zeigten jedoch mittlere oder schwere Verläufe, vier von ihnen verstarben (•Tab.2). Auch drei ältere, nur leicht an Pocken erkrankte Patienten verstarben, vermutlich bedingt durch ihre schweren Grunderkrankungen [37].

Diese Daten unterstreichen das bekannte Risiko für ungeimpfte Gesunde, aber auch ein Risiko für geimpfte Personen, die an anderen Erkrankungen leiden. Sie stimmen überein mit einer europaweiten Studie zu Pockenerkrankungen nach dem Zweiten Weltkrieg [38]. Sie zeigen aber auch, dass auch geimpfte, gesunde Personen an Pocken versterben können. Von den insgesamt 95 Personen, 83 von ihnen in Deutschland infiziert, verstarben trotz - als erfolgreich dokumentierter - Impfung vier an hämorrhagischen Pocken. In einer Studie in Madras hatten 3,4\% der Personen, die trotz Impfung erkrankten, einen fast immer tödlichen hämorrhagischen Verlauf [1]. Beigeimpften Personen sollten zusätzliche Risikofaktoren beachtet werden, so litten die beiden in Düsseldorf verstorbenen Krankenschwestern an Ekzemen und hatten eine Hepatitis in ihrer Anamnese [17]. Bei einem Ausbruch in Jugoslawien im Jahr 1972 war bei drei von sieben Personen mit letalem, hämorrhagischem Ausgang bekannt, dass sie an einer Hepatitis litten [39].

Bei der Impf- und Isolierungsstrategie ist daher zu beachten, dass beide Gruppen, Ungeimpfte, aber auch geimpfte Patienten mit anderen Erkrankungen, besonders zu schützen sind. 


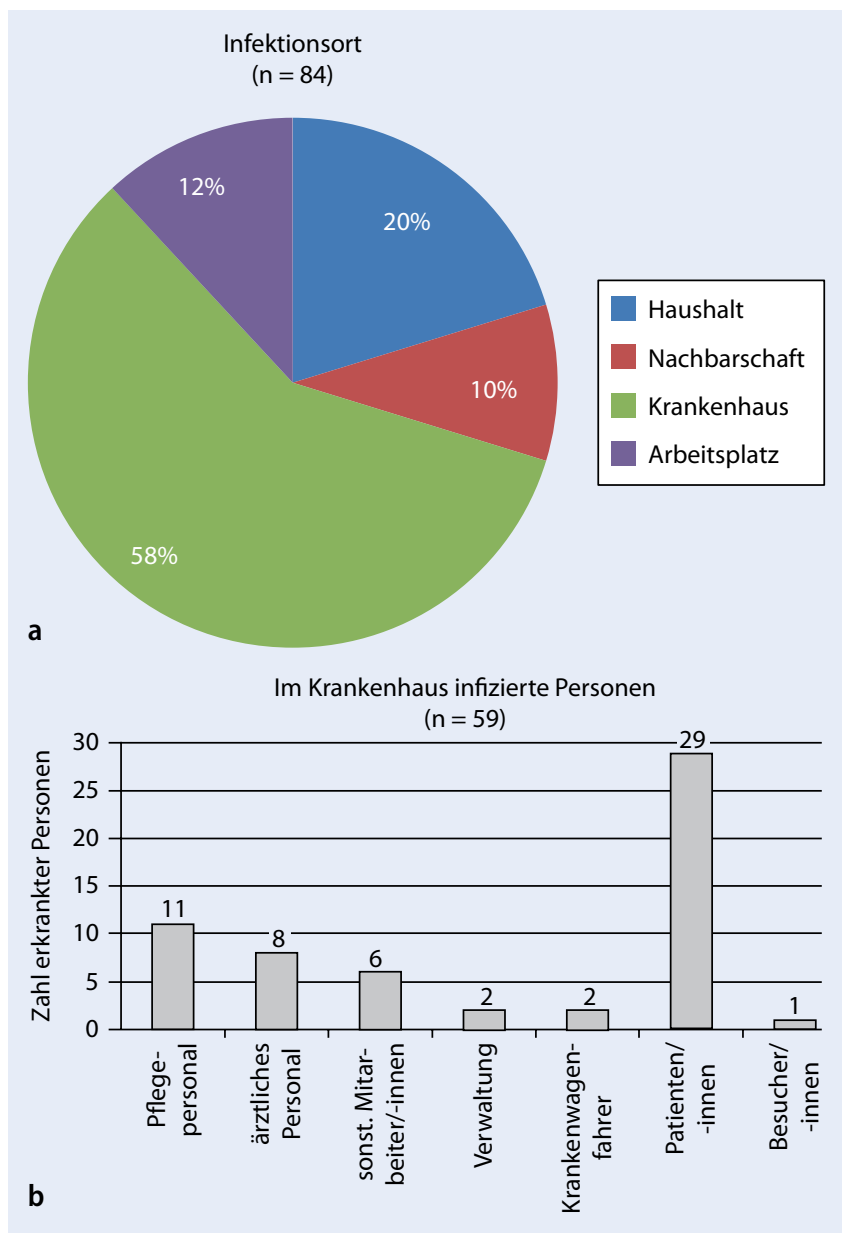

Abb. $1<$ a Umfeld, in dem die Pockenvirus-Übertragung stattgefunden hat $(n=84)$ Die Übertragungen am Arbeitsplatz erfolgten ebenfalls in den Krankenhäusern. b Aufschlüsselung der im Krankenhaus mit dem Pockenvirus infizierten Personen nach ausgeübter Tätigkeit $(n=59)$. Bei den erkrankten „sonstigen Mitarbeiter/-innen" war jeweils eine Person: Friseur, "Klinik-Chef", Medizinisch-technische Assistentin, Pförtner, Seelsorger, Schreiner

\section{Wiederimpfung, lokale Impfreaktion und Impfschutz}

Bei Pockenerkrankten wurde hier mehrfach beobachtet, dass sie auf ihre letzte Wiederimpfung nur mit Knötchen- und nicht mit einer Pustelbildung reagiert hatten. Knötchenreaktionen werden nur bei Wiederimpfungen beobachtet und sind ein Hinweis auf eine unzureichende Immunantwort. Beim Einsatz höherer Impfdosen ergaben sich auch bessere Impferfolge, begleitet von höheren Antikörpertitern. Bei einer Wiederimpfung mit einer höheren Impfdosis $\left(10^{7.7}\right.$ versus $10^{6.7} \mathrm{ID} /$ $\mathrm{ml}$ ) stieg der Anteil erfolgreicher Impfungen von 25 auf $90 \%$ [40, 41]. Im Vergleich dazu nahm die Zahl erfolgreicher Wiederimpfungen mit der Verdünnung des Impfstoffs weiter ab [42]. Eine Studie von Herrlich an 478 standardmäßig wiedergeimpften Wehrpflichtigen in Deutschland zeigt, dass 170 der 196 (87\%) Impflinge mit einer Impfpustel einen 30-fach höheren Antikörpertiter im Hämaggluti- nationstest aufwiesen. Eine Knötchenreaktion wurde bei 274 Wiederimpflingen beobachtet und blieb bei 126 (50\%) ohne signifikanten Titeranstieg, und nur $7 \%$ zeigten einen vergleichbaren Anstieg zu denen mit Pustelreaktion [43]. Als mögliche Erklärung hierfür zeigte eine weitere Studie, dass Personen mit einer Knötchenreaktion vor der Wiederimpfung im Verhältnis einen höheren Antikörpertiter hatten als andere geimpfte Personen, aber danach durchschnittlich nur einen niedrigen Anstieg der Antikörper aufwiesen [44]. Ein signifikanter Booster-Effekt konnte bei diesen Personen nur über eine höhere Impfdosis erreicht werden.

Im Gegensatz zur früher eingesetzten Impflanzette ermöglicht die auch in Deutschland für den Notfall bevorratete Bifurkationsnadel eine zuverlässigere Dosierung des Impfstoffs, mit der die Rate an Knötchenreaktionen heute erheblich sinken würde. In den Jahren 1960/61 hatten noch $63 \%$ der über 900.000 Wiederimpflinge in Deutschland nur mit einer Knöt- chenreaktion reagiert. Die Möglichkeit, dass trotz einer zurückliegenden Impfung milde Verläufe auftreten könnten, kann nicht ausgeschlossen werden [45]. Diese Annahme wird durch eine Studie an 246 Personen gestützt, deren Impfung 1388 Jahre zurücklag und die zu $97 \%$ noch einen hohen spezifischen Antikörpertiter gegen Pockenantigene aufwiesen [46]. Auch bei einigen der hier beschriebenen Pockenfälle in Deutschland lagen die letzten Impfungen teilweise über 40-50 Jahre zurück (• Tab. 1).

Knötchenbildung war auch bei den Ausbrüchen in Deutschland ein besonderes Risiko. Einerseits erkrankten mehrere geimpfte, aber nur mit Knötchenbildung reagierende Indexpatienten leicht und stellten ein Infektionsrisiko für ihre Kontakte dar, besonders weil die milde Symptomatik sie weiter am täglichen Leben teilnehmen ließ. Andererseits erkrankten drei Kontakte mit Knötchenreaktion schwer; zwei von ihnen starben: Sie betreuten trotz unzureichender Impfreaktion Kranke mit dem Vollbild der Pocken. Bei einem Pockenausbruch in Deutschland könnte es zu einigen schweren Verläufen, aber auch zu vielen milden Erkrankungen kommen, da über die Hälfte der Bevölkerung wenigstens einmal geimpft wurde. Solche Personen stellen daher ein potenzielles Risiko für eine Weiterverbreitung des Erregers dar, da die Infektion durch einen milden Verlauf möglicherweise erst spät erkannt wird.

In drei Fällen der hier aufgeführten Untersuchungen erkrankten Indexpatienten trotz einer regelrechten Impfung innerhalb des als ausreichend angesehenen Zeitraumes von drei Jahren (• Tab. 1). Einer infizierte zwei Familienangehörige, von denen einer erfolgreich, der andere erfolglos geimpft war. In diesem Fall wurde die Erkrankung erst nach 18 Tagen erkannt, wobei in der gesamten Zeit enge Kontakte zwischen den Personen bestanden [17]. Beim Management eines Ausbruchs und der Kommunikation mit der Öffentlichkeit muss daher beachtet werden, dass Personen auch bei regelrechter, erfolgreicher Impfung nicht vollständig geschützt sein können. 


\section{Übertragungsorte}

Über die Hälfte der Übertragungen $(49 / 84)$ erfolgte im Krankenhaus. Werden die Fälle in Heidelberg als Krankenhausinfektion und nicht als Infektion am Arbeitsplatz gezählt, waren es sogar $70 \%$ (59/84). In $20 \%$ der Fälle wurden Familienangehörige und in $10 \%$ Personen aus der Nachbarschaft infiziert.

Patienten im Krankenhaus und Krankenhauspersonal waren im gleichen Ausmaß mit je 29 Erkrankungen betroffen (- Abb. 1). In den in Deutschland heute vorhandenen sieben Sonderisolierstationen ist angesichts des hohen Ausbildungsgrades das Risiko einer nosokomialen Übertragung nahezu ausgeschlossen. Die Analyse der 12 Ausbrüche zeigte jedoch, dass die Indexfälle nicht als erstes bei Fachärzten oder in Spezialkliniken vorstellig werden. Es ist daher dafür Sorge zu tragen, dass die Ärzteschaft und auch Kliniken in der Peripherie Kompetenz zur Behandlung hochkontagiöser Patienten aufbauen, erhalten und pflegen, da sie damit rechnen müssen, hochkontagiöse Patienten zu versorgen, bevor diese verlegt werden können. Dies ist insbesondere von Interesse, wenn bei einem Massenanfall Infizierter, z. B. bei einem bioterroristischen Anschlag, keine Verlegung der betroffenen Personen möglich sein sollte.

\section{Fazit}

Die Analyse der Pockenausbrüche in Deutschland in der zweiten Hälfte des 20. Jahrhunderts erlaubt folgende Schlussfolgerungen, die aktuell bei den Planungen im Falle eines Pockenalarms herangezogen werden sollten:

1. Während der ersten zwei bis drei Tage nach Fieberbeginn besteht nur ein sehr geringes Übertragungsrisiko. Erst bei einer Rachenbeteiligung ist mit Kontagiosität und der Übertragung durch Aerosole zu rechnen. Aufgrund der Untersuchungsergebnisse zu asymptomatischen Kontaktpersonen ist dennoch das im Rahmenkonzept empfohlene Vorgehen sinnvoll, ab Symptombeginn mit der Rückverfolgung von möglichen Kontaktpersonen zu beginnen.
2. Die Mehrzahl der erfolgreich Geimpften ist gegen schwere Infektionsverläufe geschützt, auch wenn die Impfung lange zurückliegt. Sie können jedoch milde erkranken und eine Infektionsquelle darstellen. Todesfälle wurden auch in Einzelfällen bei erfolgreich geimpften Personen beobachtet. Dies muss sowohl bei der Impfstrategie als auch bei der Kommunikation mit der Öffentlichkeit berücksichtigt werden.

3. Wiederimpflinge mit einer Knötchenreaktion haben ein höheres Infektions- und Übertragungsrisiko als solche mit einer Impfpustel. Die Knötchenreaktion weist auf eine nur ungenügende Booster-Reaktion hin. Zeigt eine Kontaktperson nach einer Wiederimpfung nur eine Knötchenreaktion, ist besondere Aufmerksamkeit geboten - bei hohem Expositionsrisiko kann eine Quarantäne erforderlich sein.

4. Ein hoher Grad an Kompetenz bei der Ärzteschaft ist entscheidend für die schnelle Verdachtsdiagnose und die Identifizierung von Erkrankten durch Klären von Differenzialdiagnosen. Sie ist auch entscheidend für das Einleiten der notwendigen Maßnahmen im Bereich des Öffentlichen Gesundheitsdienstes zur Abriegelung des Ausbruchs.

5. Durch das Konzept der Sonderisolierstationen - mit regelmäßigem Training und den organisatorischen und baulichen Gegebenheiten - wird das Risiko für nosokomiale Infektionen durch kontagiöse, lebensbedrohliche Erkrankungen nahezu ausgeschlossen. Gleichzeitig ist es von großer Bedeutung, dass alle Krankenhäuser auf eine zeitweise Isolierung und Behandlung von hochkontagiösen $\mathrm{Pa}$ tienten vorbereitet sind.

\section{Korrespondenzadresse}

\section{Dr. J. Sasse}

Robert Koch-Institut

Nordufer 20, 13353 Berlin

SasseJ@rki.de
Danksagung. Vielen Dank an Frau Gloria CastroFrenzel für die umfassende Hand-Recherche der Literatur.

\section{Einhaltung ethischer Richtlinien}

Interessenkonflikt. J. Sasse und H.R. Gelderblom geben an, dass kein Interessenkonflikt besteht.

\section{Literatur}

1. Fenner F, Henderson DA, Arita I, Jezek Z, Ladnyi ID (1988) Smallpox and its eradication. WHO, Geneva

2. Brumfiel $G$ (2003) Russia's bioweapons labs: still out in the cold. Nature 423:678-680

3. Cdc (2014) CDC media statement on newly discovered smallpox specimens. In:CDC, http://www. cdc.gov/media/releases/2014/s0708-NIH.html. Zugegriffen: 12. Jan. 2015

4. Jackson RJ, Ramsay AJ, Christensen CD, Beaton S, Hall DF, Ramshaw IA (2001) Expression of mouse interleukin- 4 by a recombinant ectromelia virus suppresses cytolytic lymphocyte responses and overcomes genetic resistance to mousepox. J Virol 75:1205-1210

5. Gelderblom H (2003) Elektronenmikroskopie im Methodenspektrum der Bioterrorismus-Diagnostik. Bundesgesundheitsbl Gesundheitsforsch Gesundheitsschutz 46:984-988

6. Nitsche A, Ellerbrok H, Pauli G (2004) Detection of orthopoxvirus DNA by real-time PCR and identification of variola virus DNA by melting analysis. J Clin Microbiol 42:1207-1213

7. Zur G (1948) Sporadische Fälle von Variola Vera und Variolois im Februar-März 1947. Dtsch Med Wochenschr 73:195-198

8. Nauck EG (1957) Die Bedeutung der Pocken im internationalen Reiseverkehr. Bundesgesundheitsbl Gesundheitsforsch Gesundheitsschutz 3:37-38

9. Andres KH, Lieske H, Lippelt $\mathrm{H}$ et al (1958) Variola. Dtsch Med Wochenschr 83:12-17

10. Anders W (1961) Epidemiologische Besonderheiten der Pockenausbrüche im Nachkriegsdeutschland. Bundesgesundheitsbl Gesundheitsforsch Gesundheitsschutz 4:181-184

11. Wolff G (1959) Die Heidelberger Pockenepidemie. Dtsch Med Wochenschr 84:639-639

12. Anonym (1959) Pockenfälle in Heidelberg. Dtsch Med Wochenschr 84:209-210

13. Dumjahn G (1960) Epidemiologische Betrachtungen zur Pockengefährdung Europas. Dtsch Gesundheitsw 15:2435-2443

14. Daniels B (1963) Zur Rechtsprechung über die Pockenerkrankungen in Heidelberg. Öff. Gesundheitsdienst 25:106-107

15. Dumjahn G, Kima T, Henne K (1959) Ein Fall von Variolois. Dtsch Gesundheitsw 14:1649-1655

16. Herrlich A, Diesfeld HJ, Schmidt H (1961) Die Pockenerkrankungen in Ansbach 1961. Dtsch Med Wochenschr 30:1413-1420

17. Baier D (1964) Die Düsseldorfer Pockenerkrankungen 1961/62. Münch Med Wochenschr 106:19171926

18. Anders W, Posch J (1962) Die Pockenausbrüche 1961/62 in Nordrhein-Westfalen. Bundesgesundheitsbl Gesundheitsforsch Gesundheitsschutz 17:265-269

19. Freidank P, Heeger W, Spies A, Stickl H (1966) Pocken in Kulmbach. Münch Med Wochenschr 108:869-874

20. Anders W (1966) Pocken in Kulmbach. Bundesgesundheitsbl Gesundheitsforsch Gesundheitsschutz 9(2):21-22 
21. Höpken W, Willers H, Knocke K et al (1973) Pockenerkrankung im März 1967. Dtsch Med Wochenschr 98:587-593

22. Epp C, Schopp W (1967) Pocken in Regensburg. Dtsch Med Wochenschr 93:384-387

23. Wehrle PF, Posch J, Richter KH, Henderson DA (1970) An airborne outbreak of smallpox in a German hospital and its significance with respect to other recent outbreaks in Europe. Bull Wld Hlth Org/Bull Org mond Santé 43:669-679

24. Gelfand HM, Posch J (1971) The recent outbreak of smallpox in Meschede, West Germany. Am J Epidemiol 93(4):234-237

25. Kleinschmidt H, Honnef B (1971) [Protection against smallpox in hospitals]. Med Welt 39:15211523

26. Hartung J (1973) Bericht über den 2. Pockenalarm in Hannover. Der Hausarzt 24:454-455

27. Wirtz A, Gottschalk R, Weber H (2003) Management biologischer Gefahrenlagen. Bundesgesundheitsbl Gesundheitsforsch Gesundheitsschutz 46:1001-1009

28. WHO (2009) factsheets smallpox. In: http://www. who.int/mediacentre/factsheets/smallpox/en/index.html. Zugegriffen: 17. Feb. 2012

29. Henderson DA, Inglesby TV, Barlett JG et al (1999) Smallpox as biological weapon. JAMA 281(22):2127-2137

30. Sarkar JK, Mitra AC, Mukherjee MK, De SK, Mazumdar DG (1973) Virus excretion in smallpox/1. Excretion in the throat, urine, and conjunctiva of patients. Bull Wld HIth Org 48:517-522

31. Downie AW, St. Vincent LS, Meiklejohn G et al (1961) Studies on the virus content of mouth washings in the acute phase of smallpox. Bull Wld HIth Org 25:49-53

32. Sarkar JK, Mitra AC, Mukherjee MK, De SK, Mazumdar DG (1973) Virus excretion in smallpox/2. Excretion in the throats of household contacts. Bull Wid Hlth Org 48:523-527

33. Sarkar JK, Mitra AC, Mukherjee MK (1974) Duration of virus excretion in the throat of asymptomatic household contacts of smallpox patients. Indian Med Res 62 (12):1800-1803

34. Sarkar JK, Chakravarty T, Roy KM, Chakravarty NN, Poddar G, Sarkar RK (1973) Serial isolation of variola virus in the throat of household contacts of smallpox cases. Bull Calcutta Sch Trop Med 11:2123

35. Sarkar JK, Mitra AC, Mukherjee MK, De SK (1972) Variola virus in the throat of healthy contacts of smallpox cases. Bull Calcutta Sch Trop Med 10:6162

36. Pauli G, Blumel J, Burger R et al (2010) Orthopox viruses: Infections in humans. Transfus Med Hemother 37:351-364

37. Richter KH, Ippen H (1970) Der Pockenausbruch im Meschede. Arch Hyg 154:88-89

38. Mack TM (1972) Smallpox in Europe, 1950-1971. J Infect Dis 125(2):161-169

39. Kecmanovic M, Suvakovic V (1975) Der Einfluß des Impfstatus auf die klinische Pockenform und den Krankheitsausgang. Münch Med Wochenschr 117(3):87-92

40. Richter KH, Posch J (1963) Pockenschutzimpfungen beim Auftreten von Pockenerkrankungen. Bundesgesundheitsbl Gesundheitsforsch Gesundheitsschutz 6(13):6-200

41. Herrlich A, Ehrengut W, Mayr A, Munz E, Schleussing H (1965) Die Pockenschutzimpfung. In: Handbuch der Schutzimpfungen. Springer, Berlin, S60312
42. Frey SE, Newman FK, Yan L, Belshe RB (2003) Response to smallpox vaccine in persons immunized in the distant past. JAMA 289(24):3295-3299

43. Herrlich A, Adldinger H, Mobest $H$ (1965) [Morphologic and serologic evaluation of the reaction to smallpox revaccination with special reference to virus neutralizing antibodies]. Dtsch Med Wochenschr 90:2225-2229

44. Von Dostal V, Haenzel I (1965) Zur Immunität und Problematik der Pockenschutzwiederimpfung. Schweiz Med Wochenschr 95:1749-1751

45. Meier E (1964) Die Pockenschutzimpfung in der Bundesrepublik Deutschland 1960 und 1961. Bundesgesundheitsbl Gesundheitsforsch Gesundheitsschutz 7(5):7-73

46. Taub DD, Ershler WB, Janowski M et al (2008) Immunity from smallpox vaccine persists for decades: a longitudinal study. Am J Med 121:10581064 